\title{
Ethical and allocation issues in liver transplant candidates with alcohol related liver disease
}

\author{
Mai Sedki, Aijaz Ahmed, Aparna Goel \\ Division of Gastroenterology and Hepatology, Stanford University School of Medicine, Stanford, CA, USA \\ Contributions: (I) Conception and design: All authors; (II) Administrative support: None; (III) Provision of study materials or patients: None; (IV) \\ Collection and assembly of data: M Sedki; (V) Data analysis and interpretation: None; (VI) Manuscript writing: All authors; (VII) Final approval of \\ manuscript: All authors. \\ Correspondence to: Aijaz Ahmed, MD. Division of Gastroenterology and Hepatology, Stanford University School of Medicine, 750 Welch Road, Suite \\ \# 210, Stanford, CA 94304, USA. Email: aijazahmed@stanford.edu.
}

\begin{abstract}
In the past decade, alcohol-related liver disease (ALD) has become the leading indication for liver transplantation (LT) in the United States. Despite this major development, there still remains some controversy in a distinct subset of this patient population, those presenting with alcoholic hepatitis (AH). There is significant debate within the transplant community regarding acceptance criteria for patients with AH requiring LT, especially those with less than 6 months of sobriety. With that being said, LT in the setting of ALD and AH has shown an improvement in survival rates; additionally, many studies have reported that careful selection of patients with ALD has produced excellent post-transplant outcomes even if transplant occurred with less than 6 months of sobriety. In this review, we aim to discuss the ethical and allocationassociated issues that arise when considering ALD and/or AH for LT; furthermore, we delve into the history, controversies, current guidelines, and future directions of LT in this subgroup.
\end{abstract}

Keywords: Ethical considerations; liver transplantation (LT); alcohol-related liver disease (ALD)

Received: 14 January 2020; Accepted: 17 September 2020; Published: 25 July 2022.

doi: $10.21037 / \operatorname{tgh}-2020-13$

View this article at: http://dx.doi.org/10.21037/tgh-2020-13

\section{Introduction}

Alcohol-related liver disease (ALD) has become the leading indication for liver transplantation (LT) in the United States (1). A distinct subset of ALD, alcoholic hepatitis (AH) constitutes a high proportion of up to $35-40 \%$ of patients with alcohol use disorder (AUD) $(2,3)$ with high overall mortality of 40-50\%, and an increase in mortality of up to $70 \%$ in patients who are refractory to medical treatment $(4,5)$. LT in the setting of ALD and AH has demonstrated an improvement in survival rates (6). The lack of medical therapies presents a unique and controversial set of management challenges and ethical considerations. Medical criteria for the evaluation for LT for ALD are similar across the majority of transplant centers in the United States, while policies for LT in patients with AH are variable. The majority of liver transplant centers require a 6-month period of sobriety prior to consideration for LT. The validity and utility of this rule in excluding patients for LT has been questioned in the past decade given the high 6-month mortality observed in patients with $\mathrm{AH}$ and lack of evidence suggesting different outcomes in patients with extended sobriety prior to LT. There is increasing evidence that carefully selected patients with ALD have excellent post-transplant outcomes even when transplanted with less than 6 months of sobriety (7-10). What remains a challenge is the identification of patients (I) with a low likelihood to recover hepatic function with sobriety, and (II) with a low risk of alcohol relapse.

On the other hand, there has been debate regarding LT for AUD arising from the outdated perception of the "selfinflicted" nature of disease. Attitudes and practices are divided between those favoring early transplant as rescue therapy and those who believe that the risks and burden 
of this practice outweigh the benefits. The latter group argues that due to the shortage of donor organs, LT should be reserved for other "preventable" disease etiologies and that by including ALD and AH patients, this may decrease the donor pool and potentially lower public support for transplantation and organ donation. For those with ALD, the controversy has been somewhat settled over the past few decades with the implementation of the commonly used 6-month sobriety period prior to LT. More recently, there has been increasing advocacy for LT as a rescue therapeutic option for patients with $\mathrm{AH}$ refractory to medical management. In this review, we discuss the ethical and allocation issues concerning LT in ALD and AH, more specifically we explore the history, controversies, current guidelines, and future directions of LT in this subgroup while providing evidence of acceptable outcomes in this patient population.

\section{History of LT and AUD}

LT was rarely performed in ALD until 1983 when the United States National Institutes of Health Consensus Conference on Liver Transplantation concluded that ALD is an appropriate indication for LT if the subject is deemed likely to abstain from alcohol after LT (10). Following this, there was a marginal increase in the number of LTs being performed for ALD. However, it was not until Starzl et al. published their findings reporting postLT survival rates of up to $73 \%$ in ALD patients was a convincing argument in favor of LT for ALD built (11). Shortly after, the US healthcare financing administration (HFA) approved ALD as one of the indications for LT with the recommendation of a "significant" period of abstinence prior to transplantation and the availability of a reasonable social support system. The period of abstinence that was arbitrarily chosen was 6 months, which originally emerged from three poorly controlled studies but is still frequently used by most transplant centers in the US to delist patients with ALD. The role of the 6-month rule will be further discussed in coming sections. In an effort to satisfy the second condition for approval by the US HFA, Beresford et al. proposed a selection method to identify ALD patients suitable for LT (4). Furthermore, Lucey et al. proposed a multidisciplinary approach identifying psychosocial predictors of long-term sobriety and compliance after LT among patients with ALD (12). From there, ALD became a common indication for LT and since 2016 has surpassed to become the leading indication for LT in the US $(1,13,14)$. Further reports showed that most ALD patients selected for LT have similar, if not better, survival rates compared to those who underwent LT for other indications with a 1-year survival rate of $86 \%(15-19)$.

\section{AH}

$\mathrm{AH}$, a distinct entity of ALD, is a clinical syndrome of acute hepatic decompensation secondary to inflammatory liver injury in the setting of recent/abundant alcohol use. It is characterized by a progressive onset of jaundice, mild to moderate elevation in liver enzymes, and coagulopathy. The amount of alcohol intake that puts an individual at risk of AH is not known. Severity of disease is measured by Maddrey's Discriminant Function (DF); Maddrey's DF $>32$ is indicative of severe $\mathrm{AH}$ and standard medical treatment consists of high-dose corticosteroids and supportive care $(20,21)$. Response to corticosteroid therapy can be measured by the Lille score after 7 days of therapy; patients with Lille score $>0.45$ at 1 week do not benefit from continued corticosteroids (22). In this group, 3-month mortality rates range from $60-70 \%$ and as high as $90 \%$ within the first year (23-26). With the 6-month abstinence rule in place and lack of other medical treatment options, no other therapy options are available for this group. Abstinence for 6 months before LT is not an achievable goal for many patients, a reality that prompted inquiry into whether early transplantation may be feasible.

In 2011, Mathurin et al. published the first report on early LT in AH. They reported a significant decrease in mortality in patients with a favorable psychosocial profile presenting with severe AH refractory of medical therapy (23). Since then, there have been a number of cohorts reporting similar outcomes (27-29). Furthermore, studies have examined the rates of alcohol relapse, graft survival, and mortality between ALD patients transplanted with limited sobriety (less than 6 months) and greater than 6 months sobriety and found no statistically significant difference $(6,28,29)$. Furthermore, a study from the UNOS database showed that patients transplanted for $\mathrm{AH}$, when compared to those transplanted for ALD had comparable 5-year graft and patient survival (30). A systematic review of 11 studies concluded that survival and recurrence rates are similar in early transplanted AH patients and ALD patients (31). Shedding light upon such possibilities has opened up avenues not previously considered to be an option. With multiple studies providing sufficient evidence to support 
similar or improved survival rates and comparable outcomes in early transplant $\mathrm{AH}$ candidates, this has become a dire topic of concern since the mortality in this group is so high and no medical intervention has been shown to be beneficial.

\section{Origin and validity of the 6-month sobriety rule}

The 6-month rule was based off of an observational study which reported a significantly higher mortality rate (36\%) in ALD patients with less than 6 months of sobriety prior to LT, compared to $16 \%$ with greater than 6 months of abstinence (15). However, this study lacked sufficient power to draw conclusions as it examined a cohort of only 11 patients. The 6-month rule's original purpose was to provide sufficient time for potential improvement in hepatic function and portal hypertension with the possibility that the patient may no longer need LT. However, since its inception, it has been used as a surrogate marker for predictability of alcohol relapse rates (probability of longterm sobriety). With that being said, the 6 -month rule as a surrogate marker has never been shown to correlate with survival after LT nor reliably predict abstinence or compliance post-LT $(32,33)$. Various reports have shown 6-month abstinence to be a poor predictor of post transplantation abstinence $(33,34)$.

An interval of sobriety is medically justifiable as abstinence can sometimes lead to significant improvement in the complications of portal hypertension, regaining liver function to a point where LT is no longer medically required (35). However, when assessing long term sobriety, the 6-month abstinence period has been shown to be an inadequate for prediction (4,36-38). This time restriction has also inadvertently negatively impacted the referral and work-up process for potentially eligible candidates (23). In fact in the US, a large majority of candidates with cirrhosis secondary to ALD who may be eligible for LT referral are not being referred; Kotlyar et al. estimated a potential number of patients with ALD and decompensated AH to be 100,000 patients per year with only $10 \%$ of those being referred, $4 \%$ listed, and 1.2\% undergoing LT (39). Another potential negative effect of this rule, is the rise of interprogram inconsistency in criteria for LT candidacy which can further exacerbate the aforementioned discrepancy in referral for LT since providers can often be misinformed about when an ALD patient may be suitable for LT referral. The fact remains that there are no national or international mandates on sobriety length and candidacy for LT consideration. Guidelines from the American Association for the Study of Liver Diseases (AASLD), the European Association for the Study of the Liver (EASL), UNOS, and the French Consensus Conference declared the 6-month rule an obsolete absolute contraindication and should no longer be used to determine candidacy for LT (40-43).

Additionally, a potential adverse effect of the implementation of such strict sobriety rules may encourage dishonesty from the LT candidate or their support system in an attempt to advocate for the patient's listing for LT. This may in turn lead to worse LT outcomes, as patients may not receive the needed therapy for AUD pre and post transplantation. Patients may not be forthcoming with their alcohol use history and therefore an appropriate assessment and plan for the treatment of substance abuse will not be initiated, rendering this patient at a higher risk for alcohol relapse and adverse outcomes overall. Compared with other disease etiologies, ALD patients have been reported to have a higher severity of hepatic decompensation (44) and therefore, the decision to deny LT on the account of a perceived-but poorly assessed-high risk for alcohol relapse should not be taken lightly.

\section{Ethical considerations}

Donor grafts remain a scant resource despite the efforts made to increase organ donation; an estimated $15 \%$ of patients on the waitlist die before a liver becomes available (45). This clear shortage has raised doubts regarding the priority that can be allotted to patients with ALD, a condition that historically was considered to be a predictable consequence of one's own actions. This idea has long been refuted, however this punitive meritocratic ideology can still linger and impact decision making (46). The scarcity of organs for transplantation imposes the need to incorporate equity, justice, utility and benefit in the definition of priorities for organ allocation; concepts which are frequently in conflict and hard to fully reconcile. And since LT, in most cases, may be a life-saving procedure, the selection and candidacy process require the integration of these major ethical aspects. It is therefore essential for the transplant community to not only define the selection criteria for listing but do so in an equitable and fair manner. With that being said, selection criteria inevitably introduce a source of discrimination. However, if these parameters have been shown in previous studies to suggest better outcomes, then utilizing them is more reliable and equitable than eliminating a large number of patients based on non- 
evidence based parameters, such as the 6-month abstinence rule.

Historically, LT for ALD mounted controversies and widespread debate due to the idea that AUD is a selfinflicted disease with predictable consequences and therefore less "deserving" of the scarce resource of donor organs. However, AUD is no longer viewed to be a result of moral weakness and self-destructive behavior, but a chemical dependence driven by pathophysiology that has yet to be elucidated (47). Neuroscience has shown that AUD is a chronic relapsing medical disease rather than a bad habit. Ethical principles necessitate active treatment of these patients without discrimination (48-51), Therefore, AUD has to be accepted as a medical disease that, in some cases, involves a genetic predisposition (52). As AUD is a chronic disease, it is to be expected that it should persist after LT. This stresses the importance of intensive lifelong medical and psychological care for ALD LT recipients; in fact, post LT surveillance might be much more important than pre-LT selection (32). Therefore, the notion that ALD is not as valid as any other indication for LT is unethical. Furthermore, this ideology of personal responsibility for health relies on a punitive conception and it is unjust to subject only a subset of patients to this level of scrutiny. For instance, an argument could be made against patients with nonalcoholic steatohepatitis (NASH), making the choice to consume extra calories and contribute to their metabolic syndrome and therefore NASH cirrhosis. Patients with acute hepatitis B virus (HBV) infections may be blamed due to careless sexual practices yet have full access to the LT waiting list. Acute liver failure after ecstasy consumption and/or acetaminophen toxicity is also not subject to the scrutiny of ALD patients despite a potentially clearer contraindication to listing. This raises the question as to why patients with ALD or AH should be treated any differently. Moreover, various studies have reported evidence of a genetic basis for AUD, suggesting that a belief in absolute personal responsibility for ALD is speculative and unsubstantiated (53). It is an ethical requirement to provide equity in access to transplantation. A commitment to equity dictates that if and only if ALD patients are shown to have unacceptably poor transplant outcomes can they be given lower priority for transplantation (54). A number of studies have reported comparable if not superior outcomes for ALD when compared to non-ALD LT recipients including patient and graft survival $(55,56)$.

\section{The controversy}

In the past decade, there has been great controversy within the transplant community regarding early transplantation for $\mathrm{AH}$ patients. With various studies reporting positive short- and long-term outcomes in this subgroup, many providers and transplant centers have shifted the stance when approaching this topic.

\section{In favor of early transplantation}

The driving proponent of opinions in favor of early LT for $\mathrm{AH}$ are based off of two main principles discussed previously: AUD being an illness in and of itself that may be complicated by ALD and AH and the high mortality rate present in this patient subgroup with very limited treatment options and the significantly improved mortality rates in carefully selected patients with good psychosocial profiles. We previously discussed the importance of considering AUD as a chronic medical condition that may be complicated by ALD and/or AH and can similarly impact suitability for LT as diabetes mellitus or hypertension but should not disqualify patients from eligibility. Just as importantly, it should not be expected to "self-resolve" and needs to be addressed in the post LT setting with multidisciplinary teams to adequately manage and treat the underlying AUD. In terms of mortality benefit, not only does LT significantly improve survival in AH patients refractory to medical treatment but post-LT mortality and relapse rates are comparable between those transplanted for AH and chronic ALD (30,57). Positive reports from recent studies investigating early LT in severe $\mathrm{AH}$ and the lack of evidence on the utility of a pre-specified internal of abstinence prior to LT suggest that early LT should be more widely considered as a potential rescue therapy.

\section{Against early transplantation}

On the other hand, opponents of this practice argue that the limited supply of donor organs, evidence that alcohol use itself may increase the risk of death from other medical conditions (58), the fear that expansion of this practice may lead to a decrease in organ donation due to negative public opinion, and the concern of recurrent harmful drinking and therefore graft dysfunction suggest that early LT should not be more widely accepted across LT programs. In a recent 
report, it was shown that the number of ALD related LT reported has significantly increased (44). Furthermore, ALD patients on the LT waitlist had a higher severity of hepatic decompensation and higher MELD scores which may suggest that AUD and ALD patients being transplanted are at higher risk of poor outcomes.

In terms of public opinion, historically polls seemed to consistently portray a generalized resistance to transplantation for alcoholics (59), however a more recent survey suggests that public opinion may be shifting gear towards a more neutral stance in regards to the role of early LT in severe AH (60). More importantly, it is difficult to predict whether a restrictive and focused evaluation investigating early LT in AH can be consistently and reliably implemented in a wider array across LT programs (23). There is already a lack of clear evidence on which criteria are necessary to ensure adequate outcomes, this may further potentiate ethical and moral dilemmas regarding appropriate referral of such patients leaving providers in the community without clear guidelines for this practice.

\section{Outcomes: mortality, graft survival, relapse rates}

An alleged argument against LT for ALD is that graft survival rates are lower in ALD patients than in patients with cirrhosis etiologies and attribute that to relapses of AUD (17). However, it has been shown that the 5-year graft survival rate in patients with ALD compared to all other LT recipients is comparable, with an approximated 5-year relapse rate of 20-50\% (61-63). The long-term mortality of transplantation for ALD is comparable to and even lower than that for LT related to other causes (64); outcomes are superior to those with chronic viral hepatitis (65). Another major reservation against LT for ALD is the risk of alcohol relapse. Surprisingly, little evidence exists to document a significant detrimental effect of relapse on graft or patient survival. Post-transplant alcohol relapse varies from $11 \%$ to $50 \%$, although graft failure occurs in less than $17 \%$ of cases, with a mortality percentage lower than $5 \%(65)$. Of note, the wide interval is likely due to the lack of consensus on the definition of relapse, with some studies reporting slips and others considering only sustained alcohol use. Death or graft loss occurs in only about $4 \%$ of all ALD patients who experience a relapse of their disease (66). The most common causes of death in patients who continued to consume alcohol were cardiovascular events and cancer, not liver failure directly caused by alcohol consumption (64). On the other hand, a large French study examining the prevalence of recurrent ALD cirrhosis after LT reported that up to one third of patients develop recurrent ALD cirrhosis within 5 years of transplant (67).

\section{Living donor LT}

When deliberating ethical issues, living donor LT should be carefully considered. AH patients listed for deceased donor LT can also be considered for living donor LT. Despite the fact that medical risks to the potential donor are the same regardless of the recipient's disease etiology, the donor's capacity for appropriate informed consent and decision making capacity cannot be regarded as uniform across the various disease etiologies. Living liver donors undergo a considerable risk to themselves including a $40 \%$ risk of medical complication as well as the potential of psychological complications both related to procedure preparedness and post-operative implications $(68,69)$. With that being said, this risk is the same across liver organ donation for any disease etiology however what is important to denote here is the increased psychological risk these potential donors face in the case of AH. The clinical urgency of AH patients as well as the limited time available may lead to an increased pressure placed upon potential donors to decide without fully considering the implications which may render the informed consent process inadequate. It is therefore essential for the transplant program to ensure that the situational urgency is not interfering with the process of informed consent and ultimately donors' decision-making capacity.

\section{Psychosocial perspective}

The importance of a multidisciplinary team to evaluate the adequacy of potential transplant candidates cannot be stressed enough; ideally patients should be interviewed directly by both social work and mental health, preferably an addiction specialist with transplant experience. This not only assesses the suitability of the patient for LT but provides a venue to identify further treatment needs for the underlying AUD. It is indispensable to highlight the importance of anticipating further treatment needs and putting in place interventional strategies in the post-LT setting to mitigate the risk of potentially poor outcomes; this not only tackles the chronic condition that needs to be addressed but has been shown to improve overall outcomes. The multidisciplinary nature of the psychosocial transplant team is important to mention as the composition of the 
team matters and may dictate the quality and strength of recommendations to improve short- and long-term outcomes.

In the expedited evaluation that often is necessary in AH patients, psychosocial assessment relies more heavily on a stringent selection criterion as pre-LT rehabilitation is not feasible. To compliment these criteria set forth by each transplant program, psychometric scales and instruments can be utilized to assist in the integration of data and accurate identification of treatments strategies. There have been various assessment tools utilized in this setting. The Stanford Integrated Psychosocial Assessment for Transplantation (SIPAT) is a validated assessment tool that has been widely used in multiple transplant centers in assessing patients for transplantation (7). More recently, it is being studied in the severe AH setting with early LT. Additionally, collateral information from family members can often help but may be unreliable given the potentially judgmental nature of the process. To date, there has not been sufficient evidence to report on a single factor or profile that can predict recurrence of alcohol use post-LT. In a recent study, four objective pretransplant variables were used to identify $\mathrm{AH}$ candidates considered for early LT with low risk for sustained alcohol use post LT (70). Those variables included $>10$ drinks per day at initial hospitalization, multiple prior rehabilitation attempts, prior alcohol-related legal issues, and prior illicit substance abuse; all of which are part of various other pretransplant assessment tools, however utilizing these four specific variables was shown to have a high negative predictive value but lacked strength in identifying patients at high risk (70). Currently, due to the lack of sufficient experience with this novel practice, the LT field draws on predictors of relapse in ALD LT patients from the general AUD patient population Therefore, larger studies and experience is needed to expand upon this much needed topic.

\section{Conclusions}

ALD has become the leading indication for LT in the US after having been an absolute contraindication less than 50 years ago (1). This evolvement can also be seen in a unique entity of ALD, AH which previously was only managed with abstinence and medical treatment with corticosteroids. However, in the past decade multiple studies have reported positive outcomes in $\mathrm{AH}$ patients receiving rescue LT. This has raised controversy within the transplant community. In this review, we discuss the opposing viewpoints as well as the moral dilemmas that arise with this practice and ethical considerations that must be addressed when contemplating wider acceptance of this practice across LT programs.

\section{Acknowledgments}

Funding: None.

\section{Footnote}

Provenance and Peer Review: This article was commissioned by the Guest Editors (Sanjaya K. Satapathy, David Bernstein, Nitzan Roth) for the series "Liver Transplantation in NASH and ALD" published in Translational Gastroenterology and Hepatology. The article has undergone external peer review.

Conflicts of Interest: All authors have completed the ICMJE uniform disclosure form (available at https://tgh. amegroups.com/article/view/10.21037/tgh-2020-13/coif). The series "Liver Transplantation in NASH and ALD" was commissioned by the editorial office without any funding or sponsorship. The authors have no other conflicts of interest to declare.

Ethical Statement: The authors are accountable for all aspects of the work in ensuring that questions related to the accuracy or integrity of any part of the work are appropriately investigated and resolved.

Open Access Statement: This is an Open Access article distributed in accordance with the Creative Commons Attribution-NonCommercial-NoDerivs 4.0 International License (CC BY-NC-ND 4.0), which permits the noncommercial replication and distribution of the article with the strict proviso that no changes or edits are made and the original work is properly cited (including links to both the formal publication through the relevant DOI and the license). See: https://creativecommons.org/licenses/by-nc-nd/4.0/.

\section{References}

1. Cholankeril G, Ahmed A. Alcoholic Liver Disease Replaces Hepatitis C Virus Infection as the Leading Indication for Liver Transplantation in the United States. Clin Gastroenterol Hepatol 2018;16:1356-8.

2. Singal AK, Anand BS. Recent trends in the epidemiology of alcoholic liver disease. Clin Liver Dis (Hoboken) 
2013;2:53-6.

3. Lucey MR, Mathurin P, Morgan TR. Alcoholic Hepatitis. N Engl J Med 2009;360:2758-69.

4. Beresford TP, Everson GT. Liver transplantation for alcoholic liver disease: Bias, beliefs, 6-month rule, and relapse-but where are the data? Liver Transpl 2000;6:777-8.

5. Mathurin P. Corticosteroids for alcoholic hepatitis—what's next? J Hepatol 2005;43:526-33.

6. Lee BP, Mehta N, Platt L, et al. Outcomes of early liver transplantation for patients with severe alcoholic hepatitis. Gastroenterology 2018;155:422-430.e1.

7. Maldonado JR, Dubois HC, David EE, et al. The Stanford Integrated Psychosocial Assessment for Transplantation (SIPAT): A New Tool for the Psychosocial Evaluation of Pre-Transplant Candidates. Psychosomatics 2012;53:123-32.

8. Maldonado JR, Sher Y, Lolak S, et al. The Stanford Integrated Psychosocial Assessment for Transplantation. Psychosom Med 2015;77:1018-30.

9. Brown RS. Transplantation for Alcoholic Hepatitis Time to Rethink the 6-Month "Rule." N Engl J Med 2011;365:1836-8.

10. Lucey MR. Liver transplantation in patients with alcoholic liver disease. Liver Transpl 2011;17:751-9.

11. Starzl TE, Van Thiel D, Tzakis AG, et al. Orthotopic liver transplantation for alcoholic cirrhosis. JAMA 1988;260:2542-4.

12. Lucey MR, Merion RM, Henley KS, et al. Selection for and outcome of liver transplantation in alcoholic liver disease. Gastroenterology 1992;102:1736-41.

13. Smith JM, Weaver T, Skeans MA, et al. OPTN/SRTR 2016 Annual Data Report: Intestine. Am J Transplant 2018;18:254-90.

14. Guirguis J, Chhatwal J, Dasarathy J, et al. Clinical Impact of Alcohol-Related Cirrhosis in the Next Decade: Estimates Based on Current Epidemiological Trends in the United States. Alcohol Clin Exp Res 2015;39:2085-94.

15. Kumar S, Stauber RE, Gavaler JS, et al. Orthotopic liver transplantation for alcoholic liver disease. Hepatology 1990;11:159-64.

16. Bird GLA, Williams R. Treatment of advanced alcoholic liver disease. Alcohol Alcohol 1990;25:197-206.

17. Bird GL, O'Grady JG, Harvey FA, et al. Liver transplantation in patients with alcoholic cirrhosis: selection criteria and rates of survival and relapse. BMJ 1990;301:15-7.

18. McCurry KR, Baliga P, Merion RM, et al. Resource utilization and outcome of liver transplantation for alcoholic cirrhosis. A case-control study. Arch Surg 1992;127:772-6; discussion 776-7.

19. Poynard T, Barthelemy P, Fratte S, et al. Evaluation of efficacy of liver transplantation in alcoholic cirrhosis by a case-control study and simulated controls. Lancet 1994;344:502-7.

20. Mathurin P, O'Grady J, Carithers RL, et al. Corticosteroids improve short-term survival in patients with severe alcoholic hepatitis: meta-analysis of individual patient data. Gut 2011;60:255-60.

21. Rambaldi A, Saconato HH, Christensen E, et al. Systematic review: glucocorticosteroids for alcoholic hepatitis - a Cochrane Hepato-Biliary Group systematic review with meta-analyses and trial sequential analyses of randomized clinical trials. Aliment Pharmacol Ther 2008;27:1167-78.

22. Louvet A, Naveau S, Abdelnour M, et al. The Lille model: A new tool for therapeutic strategy in patients with severe alcoholic hepatitis treated with steroids. Hepatology 2007;45:1348-54.

23. Mathurin P, Moreno C, Samuel D, et al. Early liver transplantation for severe alcoholic hepatitis. N Engl J Med 2011;365:1790-800.

24. Addolorato G, Mirijello A, Leggio L, et al. Liver transplantation in alcoholic patients: impact of an alcohol addiction unit within a liver transplant center. Alcohol Clin Exp Res 2013;37:1601-8.

25. Donckier V, Lucidi V, Gustot T, et al. Reply to: "Liver transplantation and severe acute alcoholic hepatitis: An ethical consideration.” J Hepatol 2014;61:707.

26. Abenavoli L, Milic N, Rouabhia S, et al. Pharmacotherapy of acute alcoholic hepatitis in clinical practice. World J Gastroenterol 2014;20:2159.

27. Im GY, Kim-Schluger L, Shenoy A, et al. Early Liver Transplantation for severe alcoholic hepatitis in the United States-a single-center experience. Am J Transplant 2016;16:841-9.

28. Lee BP, Chen PH, Haugen C, et al. Three-year results of a pilot program in early liver transplantation for severe alcoholic hepatitis. Ann Surg 2017;265:20-9.

29. Weeks SR, Sun Z, McCaul ME, et al. Liver Transplantation for Severe Alcoholic Hepatitis, Updated Lessons from the World's Largest Series. J Am Coll Surg 2018;226:549-57.

30. Singal AK, Bashar H, Anand BS, et al. Outcomes after liver transplantation for alcoholic hepatitis are similar to alcoholic cirrhosis: Exploratory analysis from the UNOS 
database. Hepatology 2012;55:1398-405.

31. Marot A, Dubois M, Trépo E, et al. Liver transplantation for alcoholic hepatitis: A systematic review with metaanalysis. PLoS One 2018;13:e0190823.

32. Berlakovich GA. Challenges in transplantation for alcoholic liver disease. World J Gastroenterol 2014;20:8033-9.

33. DiMartini A, Day N, Dew MA, et al. Alcohol consumption patterns and predictors of use following liver transplantation for alcoholic liver disease. Liver Transpl 2006;12:813-20.

34. Foster PF, Fabrega F, Karademir S, et al. Prediction of abstinence from ethanol in alcoholic recipients following liver transplantation. Hepatology 1997;25:1469-77.

35. Alexander JF, Lischner MW, Galambos JT. Natural history of alcoholic hepatitis. II. The long-term prognosis. Am J Gastroenterol 1971;56:515-25.

36. DiMartini A, Crone C, Dew MA. Alcohol and Substance Use in Liver Transplant Patients. Clin Liver Dis 2011;15:727-51.

37. Neuberger J, Schulz KH, Day C, et al. Transplantation for alcoholic liver disease. J Hepatol 2002;36:130-7.

38. Vaillant GE. A 60-year follow-up of alcoholic men. Addiction 2003;98:1043-51.

39. Kotlyar DS, Burke A, Campbell MS, et al. A critical review of candidacy for orthotopic liver transplantation in alcoholic liver disease. Am J Gastroenterol 2008;103:73443; quiz 744.

40. United Network for Organ Sharing (UNOS). In: Minimal Criteria for Liver Transplantation. Available online: https://unos.org/wp-content/uploads/unos/Liver_patient. pdf, accessed June 28, 2019.

41. Testino G, Leone S, Sumberaz A, et al. Liver transplantation in alcoholic patients. Alcohol Clin Exp Res 2014;38:1800-2.

42. T European Association for the Study of the Liver. Electronic address: easloffice@easloffice.eu; European Association for the Study of the Liver. EASL Clinical Practice Guidelines: Management of alcohol-related liver disease. J Hepatol 2018;69:154-81.

43. Singal AK, Bataller R, Ahn J, et al. ACG clinical guideline: Alcoholic liver disease. Am J Gastroenterol 2018;113:175-94.

44. Cholankeril G, Gadiparthi C, Yoo ER, et al. Temporal trends associated with the rise in alcoholic liver diseaserelated liver transplantation in the United States. Transplantation 2019;103:131-9.

45. McMaster P. Transplantation for alcoholic liver disease in an era of organ shortage. Lancet 2000;355:424-5.

46. Artru F, Louvet A, Mathurin P. Liver transplantation for patients with alcoholic hepatitis. Liver Int 2017;37:337-9.

47. Marroni CA, Bona S, Fleck Junior AM, et al. Clinical and experimental alcoholic liver disease. J Liver Clin Res 2016;3:1028.

48. Marroni CA. Management of alcohol recurrence before and after liver transplantation. Clin Res Hepatol Gastroenterol 2015;39:S109-S114.

49. Weinrieb RM, Van Horn DHA, McLellan AT, et al. Interpreting the significance of drinking by alcoholdependent liver transplant patients: Fostering candor is the key to recovery. Liver Transpl 2000;6:769-76.

50. Tandon P, Goodman KJ, Ma MM, et al. A Shorter Duration of Pre-Transplant Abstinence Predicts Problem Drinking After Liver Transplantation. Am J Gastroenterol 2009;104:1700-6.

51. Zalewska K. NHS Organ Donation, Liver Advisory Group Alcohol Guidelines; 2017. Available online: http://www. odt.nhs.uk/pdf/non_compliance_with_selection_and_ allocation_policies.pdf, accessed June 28, 2019.

52. Ferraguti G, Pascale E, Lucarelli M. Alcohol addiction: a molecular biology perspective. Curr Med Chem 2015;22:670-84.

53. Goldman D, Oroszi G, Ducci F. The genetics of addictions: uncovering the genes. Nat Rev Genet 2005;6:521-32.

54. Ubel PA. Transplantation in alcoholics: Separating prognosis and responsibility from social biases. Liver Transpl Surg 1997;3:343-6.

55. Lucey MR, Schaubel DE, Guidinger MK, et al. Effect of alcoholic liver disease and hepatitis $\mathrm{C}$ infection on waiting list and posttransplant mortality and transplant survival benefit. Hepatology 2009;50:400-6.

56. Lucey MR. Liver transplantation for alcoholic liver disease. Nat Rev Gastroenterol Hepatol 2014;11:300-7.

57. Wells JT, Said A, Agni R, et al. The impact of acute alcoholic hepatitis in the explanted recipient liver on outcome after liver transplantation. Liver Transpl 2007;13:1728-35.

58. Westman J, Wahlbeck K, Laursen TM, et al. Mortality and life expectancy of people with alcohol use disorder in Denmark, Finland and Sweden. Acta Psychiatr Scand 2015;131:297-306.

59. Neuberger J, Adams D, MacMaster P, et al. Assessing priorities for allocation of donor liver grafts: Survey of public and clinicians. BMJ 1998;317:172-5.

60. Stroh G, Rosell T, Dong F, et al. Early Liver 
Transplantation for Patients With Acute Alcoholic Hepatitis: Public Views and the Effects on Organ Donation. Am J Transplant 2015;15:1598-604.

61. Bellamy CO, DiMartini AM, Ruppert K, et al. Liver transplantation for alcoholic cirrhosis: long term followup and impact of disease recurrence. Transplantation 2001;72:619-26.

62. Jain A, Reyes J, Kashyap R, et al. Long-term survival after liver transplantation in 4,000 consecutive patients at a single center. Ann Surg 2000;232:490-500.

63. Mackie J, Groves K, Hoyle A, et al. Orthotopic liver transplantation for alcoholic liver disease: a retrospective analysis of survival, recidivism, and risk factors predisposing to recidivism. Liver Transpl 2001;7:418-27.

64. Testino G, Burra P, Bonino F, et al. Acute alcoholic hepatitis, end stage alcoholic liver disease and liver transplantation: an Italian position statement. World J Gastroenterol 2014;20:14642-51.

65. Lim JK, Keeffe EB. Liver transplantation for alcoholic liver disease: Current concepts and length of sobriety.

doi: $10.21037 /$ tgh-2020-13

Cite this article as: Sedki M, Ahmed A, Goel A. Ethical and allocation issues in liver transplant candidates with alcohol related liver disease. Transl Gastroenterol Hepatol 2022;7:26.
Liver Transpl 2004;10:S31-S38.

66. Obed A, Stern S, Jarrad A, et al. Six month abstinence rule for liver transplantation in severe alcoholic liver disease patients. World J Gastroenterol 2015;21:4423-6.

67. Dumortier J, Dharancy S, Cannesson A, et al. Recurrent alcoholic cirrhosis in severe alcoholic relapse after liver transplantation: A frequent and serious complication. Am J Gastroenterol 2015;110:1160-6.

68. Gordon EJ, Daud A, Caicedo JC, et al. Informed consent and decision-making about adult-to-adult living donor liver transplantation: a systematic review of empirical research. Transplantation 2011;92:1285-96.

69. Abecassis MM, Fisher RA, Olthoff KM, et al. Complications of living donor hepatic lobectomy--a comprehensive report. Am J Transplant 2012;12:1208-17.

70. Lee BP, Vittinghoff E, Hsu C, et al. Predicting Low-Risk for Sustained Alcohol Use After Early Liver Transplant for Acute Alcoholic Hepatitis: The SALT Score. Hepatology 2019;69:1477-87. 